\title{
X-ray tracing using Geant4
}

\author{
E. J. Buis, G. Vacanti* \\ cosine Science 63 Computing BV, Niels Bohrweg 11, 2333 CA Leiden, The Netherlands
}

\begin{abstract}
We describe an extension to the Geant4 software package that allows it to be used as a general purpose X-ray tracing package. We demonstrate the use of our extension by building a model of the X-ray optics of the X-ray observatory XMM-Newton, calculating its effective area, and comparing the results with the published calibration curves.

Key words: X-ray optics, X-ray telescopes, Geant4, ray tracing, grazing angle scattering, XMM-Newton
\end{abstract}

PACS: 42.15.Dp, 95.55.Ka, 41.50.+h, 07.85.Fv, 78.70Ck

\section{Introduction}

The Geant4 simulation toolkit is a software library for high-energy physics developed by an international collaboration under the lead of CERN [1]. Aiming at supporting the simulation and analysis of data collected by the Large Hadron Collider, Geant4 has found a keen following in many other areas where the ability to model complex geometries and the interaction of particles and radiation with matter plays a prominent role. The open nature of the toolkit, and the large libraries of geometrical models and physical processes that are available make it easy to use and extend.

The importance of detailed ray-tracing simulations in the development, construction, testing, and operation of X-ray optics has long been recognized. First

\footnotetext{
*Corresponding author. Tel. +31 71 5284962; fax. +31 715284963.

Email address: g.vacanti@cosine.nl (G. Vacanti)

$U R L$ : cosine.nl (G. Vacanti)
}

Preprint submitted to Nuclear Instruments and Methods A

November 12, 2018 
described by Wolter in 1952 [2], the X-ray optics that would later be given his name were quickly adopted as the preferred configuration for X-ray imaging systems [3]. In 1972, Chase and Van Speybroeck [4] carried out the first numerical evaluation of the optical properties of Wolter optics. Since then several authors have investigated X-ray imaging systems (see for instance [5] and references therein).

To a large extent X-ray imaging systems can be treated as any other optical system, and their properties in terms of aberrations and focusing capabilities can be examined with the standard tools of optics.

However, when detailed performance predictions of the effective area and the point spread function are required, specific ray-tracing tools are needed in order to take into account the change of the reflectivity with the angle of incidence, and the scattering caused by the details of the surface finish. To the best of our knowledge, and certainly within the domain of X-ray optics for astrophysical missions, this problem has always been solved with ad hoc tools built to address issues related to the particular design under consideration.

We have developed a set of extensions for the Geant 4 toolkit that allows one to trace X-ray optics of arbitrary complexity, as long as the geometrical model of the system can be described by the geometry library available in Geant4. The core component of these extensions consists of a Geant4-compatible implementation of the reflection of X-ray photons on a surface. Additionally, by making use of the available Geant4 functionality, we have created a framework that allows one to introduce a microscopic description of the properties of the surfaces of the optics. Through this framework it becomes possible to model the effects that these microscopic properties have on the scattering of the photons.

Besides introducing the notion of a generic tracer for X-ray optics, this development has the potential to ease the interaction of scientists and engineers during the study phase of X-ray space missions. Geant4-based models are in fact already widely used to study the effect of cosmic radiation on the spacecraft structures and instruments. By making use of the same tools in order to model both the spacecraft and the telescope, the study of a mission can be carried out 
on the basis of a shared understanding of its mechanical and optical properties.

This article is organized as follows. In $\S 2$ we briefly describe the physics of X-ray reflection and scattering at grazing angles. In $\S 3$ the X-ray oriented extensions to the Geant4 toolkit that we have implemented are described. We proceed then to describe a sample application of our extensions in $\S 4$. Finally, in $\S$ we draw some conclusions and make some final remarks.

\section{The Reflection of X-Rays}

The reflection of X-rays at the boundary between vacuum and a medium (see Fig. 10 is described by the standard Fresnel equations (see for instance [6]). Here, in keeping with the usage in the field, we use energy instead of wavelength.

For a photon with grazing incidence angle $\theta_{i}$ and energy $\mathrm{E}$, the reflection coefficient $R$ and the transmission coefficient $T$ (both for the orthogonal and parallel polarizations) are:

$$
\begin{aligned}
R\left(\theta_{i}, E\right) & =\left|\frac{\sin \theta_{i}-n(E) \sin \theta_{t}}{\sin \theta_{i}+n(E) \sin \theta_{t}}\right|^{2} \\
T\left(\theta_{i}, E\right) & =\left|\frac{2 \sin \theta_{i}}{\sin \theta_{i}+n(E) \sin \theta_{t}}\right|^{2},
\end{aligned}
$$

where $n(E)$ is the medium's index of refraction. For different materials $n(E)$ can be obtained from various sources. In practice, the reflectivity is calculated using experimentally determined optical constants that are valid in the X-ray regime. However, when these optical constants are not available because of the lack of measurements (in the case of non standard materials) or because the photon energy is in the gamma-ray regime, where only a few optical constants are known, one can construct the optical constants using the atomic form factors (e.g. [7]).

The reflection coefficient given in Eq.(1) is valid in the case of an ideal, perfectly smooth surface. For a real surface, some fraction of the reflected photons will be scattered away from the specular direction. Under assumptions generally true for X-ray optics, it is customary to characterize a surface by its micro-roughness $\sigma$, and decrease the reflectivity by the factor 




Figure 1: Schematic representation of the reflection and transmission at the boundary between vacuum and material.

$$
\exp \left(-\left(\frac{4 \pi \sigma \sin \theta_{i}}{\lambda}\right)^{2}\right)
$$

In the context of astrophysical applications of X-ray optics, Eq.(2) is sufficient to perform calculations related to the effective area of a particular optical design. More complex calculations aiming at the prediction of the point spread function must use a more extensive theory (for instance [8], [9]), whose details are affected by the type of surface data available (e.g., surface interferograms, profilometry measurements, atomic force telescope data). Our software implementation provides a generic interface for the application of any scattering model, as is described in the following section.

\section{X-Ray-Oriented Extensions of the Geant4 Toolkit}

The extension of the Geant4 toolkit with the physics of grazing angle scattering is realized through the implementation of three classes:

G4XrayRefractionIndex This is an auxiliary class that manages the refraction index data for a particular material. 
G4XraySurface As mentioned in the previous section, the details of the interaction of X-rays on a surface can be described by scattering models of various complexity, and are also driven by the type of data describing the microscopic surface details. It is therefore not surprising that even within the same model different surfaces may have different X-ray behaviours: for instance, an X-ray mirror will have one reflecting side, and a back side treated so as to absorb or scatter away from the main beam all photons that might fall on it. Also, when assembling an optical system for which disparate surface data types are available, it may be desirable to be able to assign different scattering models to different surfaces. The class G4XraySurface provides a generic interface to do exactly this. It can be used with the standard Geant4 mechanisms to define either a logical boundary surface, or a logical skin surface. We have written sample classes that implement Fresnel reflection as described in the previous section, and that perturb the surface normal at the interaction point according to a Gaussian distribution. In general, G4XraySurface can be used to implement any scattering model appropriate for the particular situation considered.

G4XrayGrazingAngleScattering This class models a new Geant4 boundary process, and is applicable to any photon (the Geant4 particle gamma: no new particle needs to be defined). The process can compete with any other Geant4 processes that might be defined in the simulation (for instance photo-electric effect, or Compton scattering). When the Geant4 tracking system detects that a photon is crossing the boundary between two volumes (normally from vacuum to a material), this process is invoked. In turn, the process queries the surface being crossed to see if it is an Xray surface: if this is the case, the process delegates the actual interaction details to the surface. The scattered/reflected photon is handed back to the process who decides its fate - absorbed or transmitted, reflected, or propagated further-, and eventually hands the control back to the Geant4 


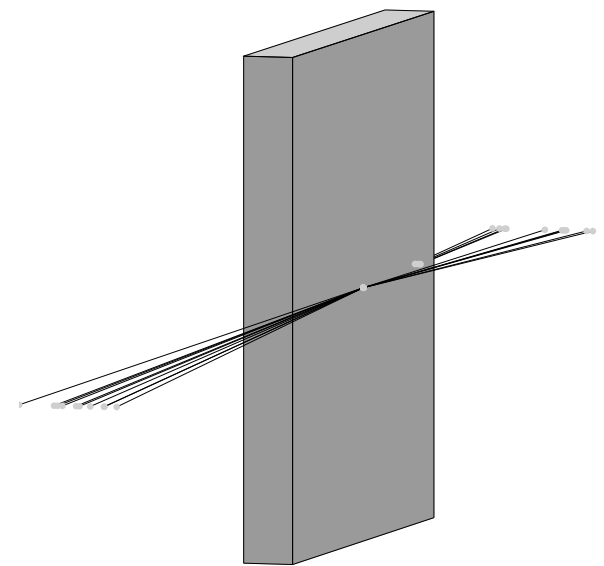

(a)

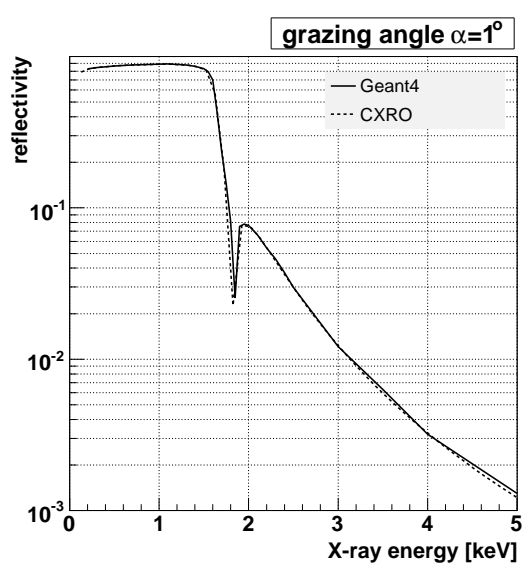

(b)

Figure 2: (a) Simulated reflectometry setup. (b) The reflectivity determined from the simulation is compared with data retrieved from the Center for X-ray Optics [10].

tracking manager. The process can be activated so as to log the details of every X-ray interaction: the coordinates of the interaction point, the volume on which the interaction has taken place, the (possibly modified) local surface normal, incoming and outgoing directions of the photon, and the reflectivity used. This information, that is commonly used in the field to understand the details of the geometrical model and how certain features on the focal plane are formed, can be accessed using the standard track information facilities of Geant4.

Using the extension a simple reflectometry set up can be constructed consisting of a single silicon plate as shown in Fig. 2(a). The reflectivity for a fixed angle of incidence can then be determined, and compared with the data obtained using the tools made available by the Center for X-Ray Optics [10], a standard reference in the field. In Fig. 2(b) the two data sets are shown to be in very good agreement. 


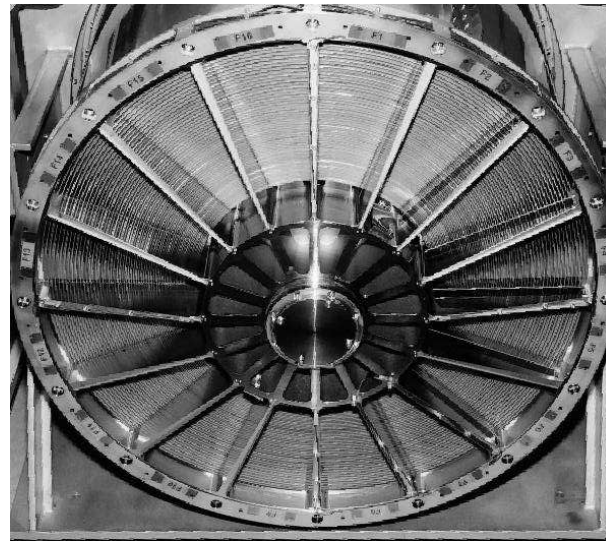

(a)



(b)

Figure 3: (a) Photograph of the front view of the XMM-Newton telescope (courtesy of the European Space Agency). (b) The Geant4 model used for the comparisons.

\section{Application Example}

As an example we have modeled the X-ray optical system of the XMMNewton telescope. Each of the three mirrors aboard XMM-Newton comprises 58 concentric mirror shells in a Wolter-I configuration, where each mirror shell consists of polished, confocal parabolic and hyperbolic surfaces. The parabolic and hyperbolic solids that are included in the Geant4 package are not directly useful to construct a Wolter-I system, because they are filled solids. We therefore approximated them with many consecutive short conical shell segments with the appropriate slope. The front view of the telescope is shown in Fig. 3 (a). In this photograph the nest of mirror shells can clearly be seen together with the spiderlike mechanical support structure. The total aperture ranges from $150 \mathrm{~mm}$ to $350 \mathrm{~mm}$. In Fig. 3(b) the same geometry is shown as implemented in our model. The mirror shells are coated with a gold layer and we assume a surface roughness of $0.5 \mathrm{~nm}$ root-mean-square.

In Fig. 4 we show the focal spot for an on-axis X-ray beam in comparison to the focal spot for an off-axis beam. The spot for the off-axis beam lies away from the center of the image plane as expected. For an off-axis angle of $2 \cdot 10^{-2}$ degrees 


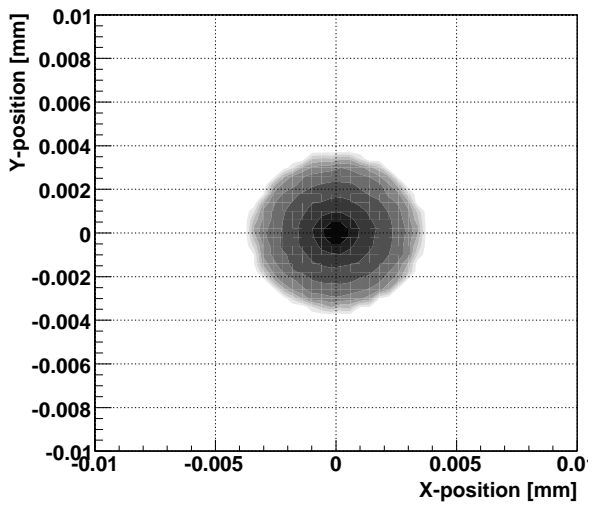

(a)

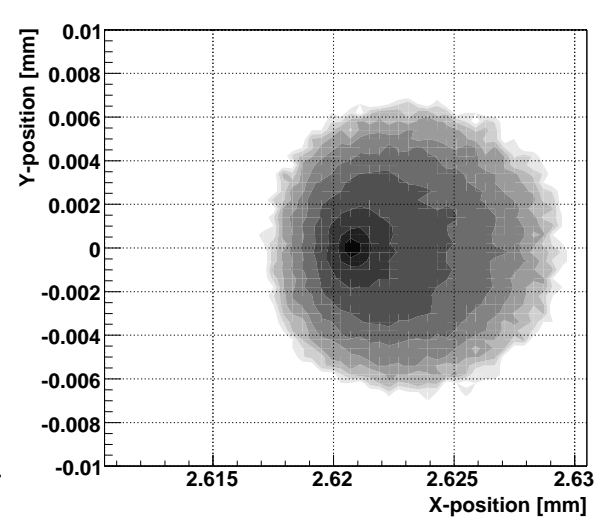

(b)

Figure 4: (a) Focal spot of the XMM-Newton telescope for an on-axis point source. (b) Focal spot for an off-axis (0.02 degrees) point source.

and a focal length of $7500 \mathrm{~mm}$, the $\mathrm{x}$-coordinate is expected to be $2.618 \mathrm{~mm}$, in agreement with the simulated result of $2.622 \mathrm{~mm}$. Note further that the shape of the spot becomes asymmetric due to coma, as expected.

By Monte Carlo simulation we have determined the effective area of the XMM-Newton optics, by taking the blocking effect due to the mechanical support structure into account. We have not taken into account any other effects, like dust contamination, or the exact form of the mirror shells. In Fig. 5 we compare our results with the ground calibration measurements reported in [11]: our results are in very good agreement with the data, differing no more than $5 \%$ from them.

\section{Conclusions}

We have created an extension of the Geant4 toolkit that makes it suitable as a platform with which to realize generic X-ray tracers. Besides modelling the grazing angle reflection of X-rays on surfaces, our extension can be used to model the effects of surface finish on the scattering of radiation.

We have demonstrated the new capabilities of the toolkit by modelling one 


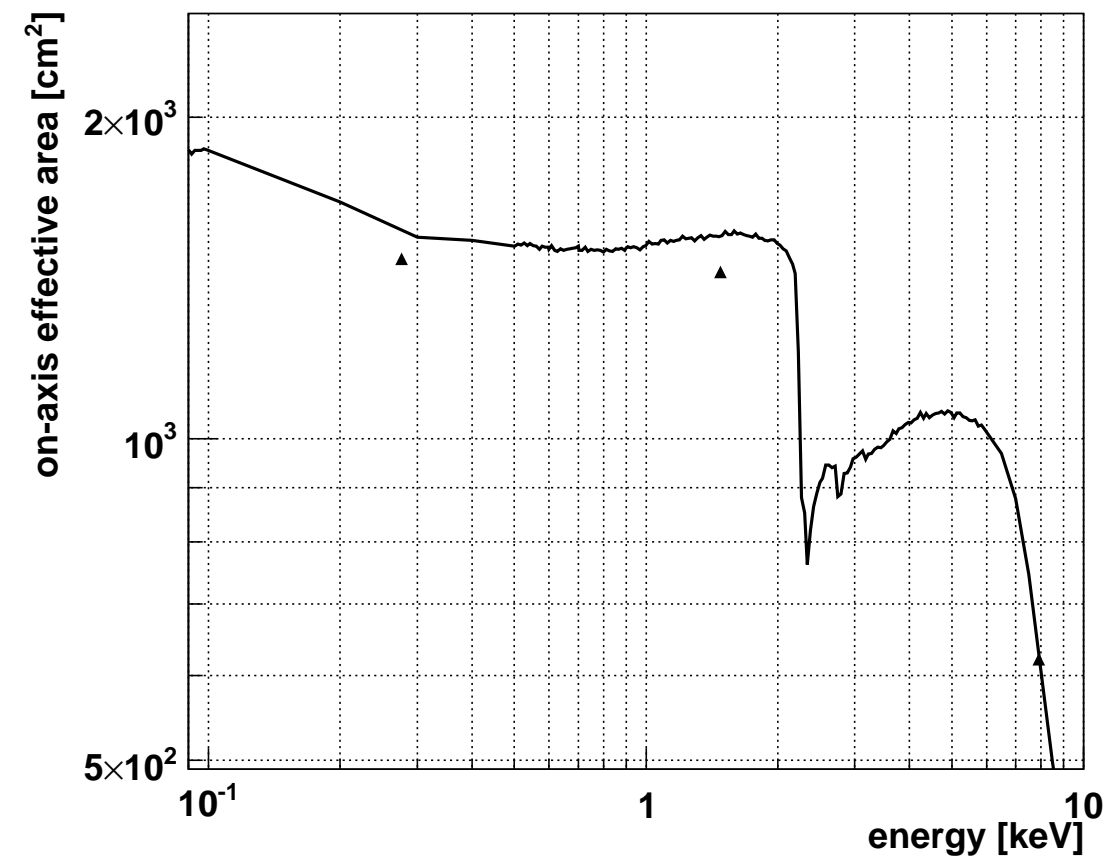

Figure 5: On-axis effective area of the XMM-Newton telescope. The solid line shows the results of our simulation. The three data points (triangles) are taken from [11]. The difference between measured and simulated effective area is less than $5 \%$. 
of the Wolter-I mirrors used on the European Space Agency's X-ray observatory XMM-Newton. Without having access to the detailed metrological data that were used during the calibration of XMM-Newton, we are able to reproduce the measured effective area to about $5 \%$. We have also shown that the main properties of the point spread function are well reproduced. Being aware of the efforts that went into accurately modelling the response of the XMM-Newton

mirrors [12], we consider our result as a demonstration of the potential our work has to ease the design and calibration of future X-ray optics.

With this extension we have turned Geant4 into a versatile modeler for X-ray optics.

\section{Acknowledgments}

This work was carried out under contract to the European Space Agency.

\section{References}

[1] Geant4 Collaboration, S. Agostinelli, et al., Nucl. Instr. and Meth. A 506 (2003) 250-303.

[2] H. Wolter, Ann. Physik 10 (1952) 94-114.

[3] R. Giacconi, B. Rossi, J. Geophys. Res. 65 (1960) 773-775.

[4] L. P. Vanspeybroeck, R. C. Chase, Applied Optics 11 (1972) 440-445.

[5] T. T. Saha, W. Zhang, Applied Optics 42 (22) (2003) 4599-4605.

[6] M. Bohr, E. Wolf, Principles of Optics, $7^{\text {th }}$ Edition, Cambridge University Press, 2005.

[7] B. L. Henke, E. M. Gullikson, J. C. Davis, Atomic Data and Nuclear Data Tables 54 (1993) 181-342.

[8] P. Beckman, A. Spizzichino, Scattering of Electromagnetic Waves from Rough Surfaces, Elsevier, 1963. 
[9] P. Croce, L. Névot, Rev. Phys. Appl. (1976) 113.

[10] Center for X-ray Optics, http://www-cxro.lbl.gov/.

[11] P. Gondoin, B. R. Aschenbach, M. W. Beijersbergen, R. Egger, F. A. Jansen, Y. Stockman, J.-P. Tock, in: R. B. Hoover, A. B. Walker (Eds.), Proc. SPIE Vol. 3444, 290-301

[12] M. Beijersbergen, Private communication (2008). 


\section{List of Figures}

1 Schematic representation of the reflection and transmission at the boundary between vacuum and material. . . . . . . . . . . 4

2 (a) Simulated reflectometry setup. (b) The reflectivity determined from the simulation is compared with data retrieved from the Center for X-ray Optics [10]. . . . . . . . . . . 6 6

3 (a) Photograph of the front view of the XMM-Newton telescope (courtesy of the European Space Agency). (b) The Geant4 model used for the comparisons. . . . . . . . . . . . . 7

4 (a) Focal spot of the XMM-Newton telescope for an on-axis point source. (b) Focal spot for an off-axis (0.02 degrees) point source.

5 On-axis effective area of the XMM-Newton telescope. The solid line shows the results of our simulation. The three data points (triangles) are taken from [11]. The difference between measured and simulated effective area is less than $5 \%$. . . . . . . 9 